\title{
FÉVRIER 1958
}

\section{REVUE}

\section{INTERNATIONA LE}

DE LA

\section{CROIX-ROUGE}

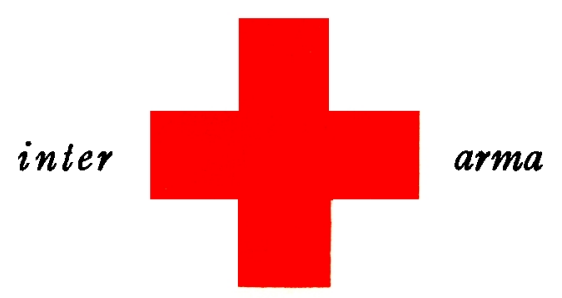

caritas

COMITE INTERNATIONAL DE LA CROIX-ROUGE GENEVE 


\title{
COMITÉ INTERNATIONAL DE LA CROIX-ROUGE
}

\author{
à Genève
}

MM. MAX HUBER, docteur en droit, ancien président de la Cour permanente de justice internationale president d'honneur (1923) 1

LÉOPOLD BOISSIER, docteur en droit, professeur honoraire de l'Université de Genève, ancien secrétaire général de l'Union interparlementaire, président (1946)

JACQUES CHENEVIĖRE, docteur ès lettres b. c. (1919)

M11 LUCIE ODIER, ex-chef du Service des infirmières-visiteuses de la Section genevoise de la CroixRouge suisse (1930)

MM. CARL J. BURCKHARDT, docteur en philosophie, ancien ministre de Suisse en France (1933)

MARTIN BODMER; docteur en philosophie h. c., vice-président (1940)

ERNEST GLOOR, docteur en méderine (1945)

PAUL CARRY, docteur en droit, professeur à l'Université de Genève (1946)

PAUL RUEGGER, ancien ministre de Surisse en Italie et en Grande-Bretagne, membre de la Cour Permanente d'Arbitrage (1948)

HENRI GUISAN, général, ancien commandant en chef de l'armée suisse (1948)

ALFREDO VANNOTTI, docteur en médecine, professeur à l'Université de Lausanne (1949)

RODOLFO OLGIATI; ancien directeur du Don suisse (1249)

M"10 MARGUERITE VAN BERCHEM, ancien chef de service de l'Agence centrale des prisonniers de guerre (1051)

MM. FRÉDÉRIC SIORDET, avocat, conseiller du Comité international de la Croix-Rouge de 1943 à 1051, vice-président (1951)

MARCEL JUNOD, docteur en médecine, délégué đu Comité international de la Croix-Rouge de 1935 a 1946 (1952)

GUILLAUME BORDIER, ingénieur dịpl. E.P.F., M.B.A. Harvard, banquier (1055)

\section{Direction.}

MM. ROGER GALLOPIN, docteur en droit, directeur exécutif

JEAN S. PICTET, docteur en droit, directeur des Affaires générales

EDOUARD de BONDELI, sous-directeur, Services financiers et administratifs

CLAUDE PILLOUD, sous-directeur, Service juridique

1 Les années indiquées dans les parenthèses désignent les dates de nomination des membres du Comité international. 


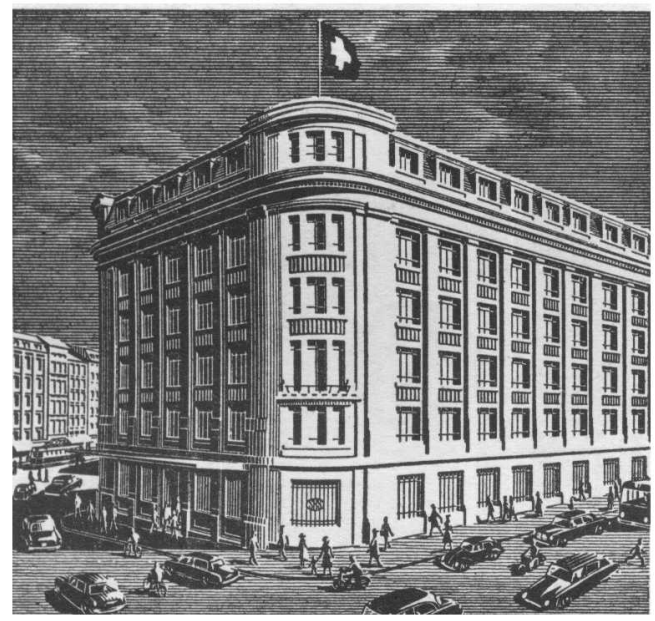

\section{SOCIETE DE \\ BANQUE SUISSE} SCHWEIZERISCHER BANKVEREIN SOCIETA DI BANCA SVIZZERA SWISS BANK CORPORATION

\section{GEN È VE}

BALE BIENNE LA OHAUX-DE-FONDS LAUSANNE NEUCHATEL ST-GALL SCHAFFHOUSE ZURICH LONDRES NEW-YORK

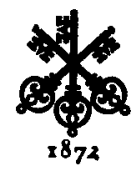

CAPITAL ET RÉSERVES FRS 275 MILLIONS 


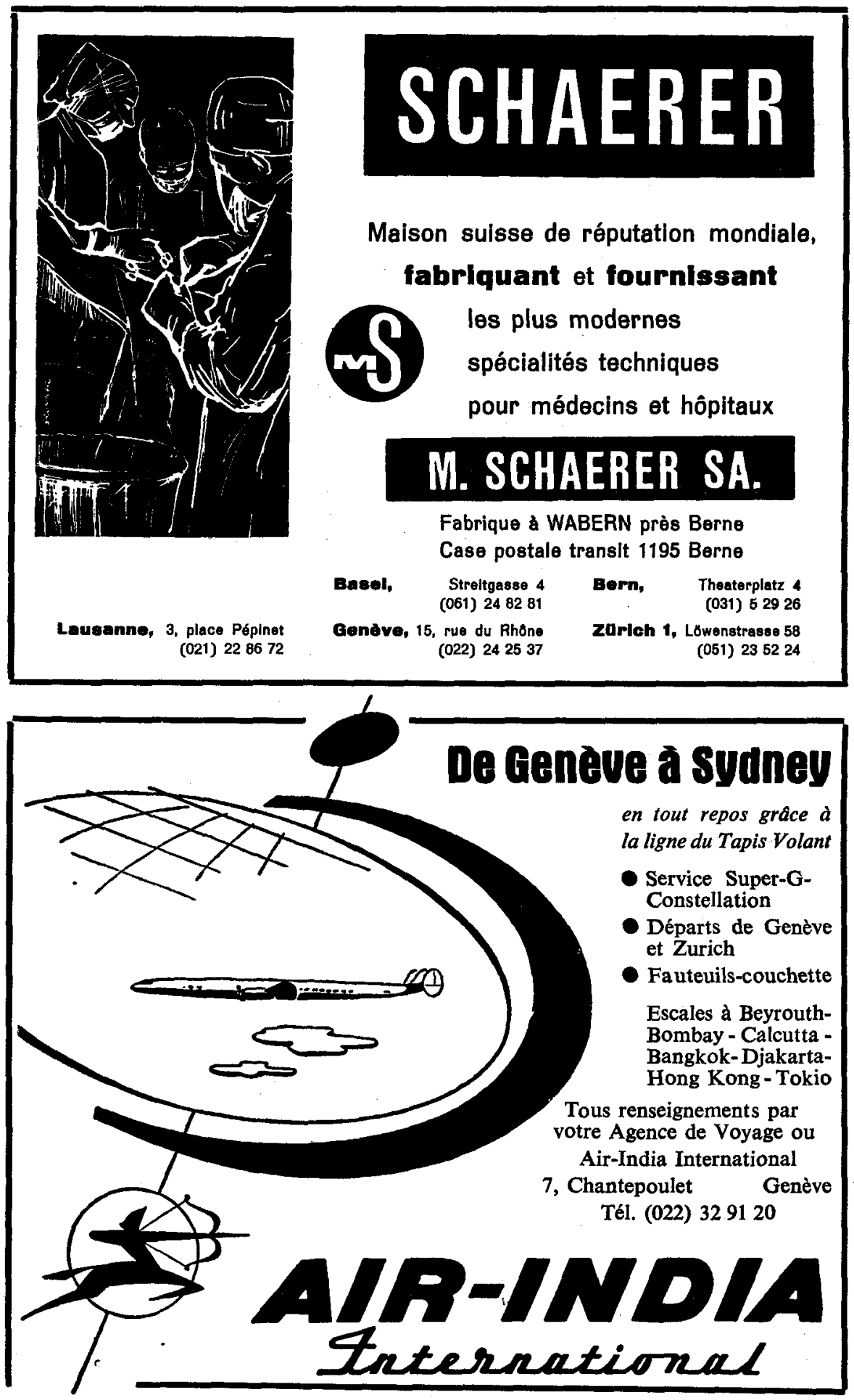




\title{
REVUE INTERNATIONALE DE LA CROIX-ROUGE
}

\author{
QUARANTIEME ANNEE - N०470 \\ FÉVRIER 1958
}

\section{SOMMAIRE}

Général H.-J. van der Giessen : Les méthodes de réadaptation appliquées par le Service de santé militaire . . . . . . . . . . . 6 I

\section{COMITÉ INTERNATIONAL DE LA CROIX-ROUGE}

Deux missions du Comité international en Asie et en Océanie 70 $V$ isite de prisonniers français par un délégué du Comité international. .. . . . . . . . . . . . . . . . . 84

Secours aux réfugiés algériens en Tunisie. . . . . . . . . . . 85

Aide aux réfugiés algériens en Afrique du Nord . . . . . . . . 86

Publications éditées par le CICR en I957 . . . . . . . . . . . 87

\section{CROIX-ROUGE INTERNATIONALE}

$X I X^{\mathrm{e}}$ Conférence internationale de la Croix-Rouge: Discours prononcés à la séance d'ouverture . . . . . . . . . .

\section{NOUVELLES DE SOCIÉTÉS NATIONALES}

Irak . . . . . . . . . . . . . . . I 106

Pologne.................. . . I07

\section{BIBLIOGRAPHIE}




\title{
SUPPLEMENTS DE LA REVUE
}

\author{
EN LANGUE ANGLAISE
}

The XIXth International Red Cross Conference. A few Thoughts on the Conference (L. Boissier). - The work of the International Humanitarian Law Commission (J. S. Pictet). - Resolutions (II).

EN LANGUE ESPAGNOLE

La XIX Conferencia Internacional de la Cruz Roja. Algunas reflexiones (L. Boissier). - Los trabajos de la Comisión del derecho humanitario (J. S. Pictet). - Resoluciones (II).

\section{EN LANGUE ALLEMANDE}

Die XIX. internationale Rotkreuzkonferenz. Ansprache des Präsidenten des Internationalen Komitees vom Roten Kreuz. - Einige Betrachtungen (L. Boissier). - Die Arbeiten der Kommission für humanitäres Recht (J. S. Pictet).

\section{LA}

REVUE INTERNATIONALE DE LA CROIX-ROUGE est publiée chaque mois par le Comité international de la Croix-Rouge

7, avenue de la Paix, Genève (Suisse) - Compte de chèques postaux I. 1767 Abonnement un an : Fr. 20,- ; le numéro Fr. 2,-

Pour les pays suivants : Algérie, Allemagne, Autriche, Belgique, Danemark, Finlande, France, Italie, Luxembourg, Maroc, Norvège, Pays-Bas, Portugal, Sarre, Suede, Tunisie, Vatican, on peut s'abonner auprès des bureaux de poste (Fr. s. 20,50).

Rédaction : JEAN-G. LOSSIER 